\title{
HOPE-3: ¿Minipolipíldora para la prevención primaria de eventos cardiovasculares en personas de riesgo intermedio?
}

\author{
HOPE-3: Mini Polypill for primary prevention of cardiovascular events in persons at intermediate risk?
}

S. Yusuf, N Engl J Med 2016; 374:2009-43

\section{Objetivos}

Evaluar el uso de la minipolipíldora (rosuvastatina 10mg + candesartán $16 \mathrm{mg}$ + hidroclorotiazida |HCTZ] 12,5mg) como terapia antihipertensiva e hipolipemiante para la prevención primaria de eventos cardiovasculares mayores en la población con riesgo cardiovascular intermedio.

\section{Diseño, lugar y pacientes}

Ensayo clínico aleatorizado, doble ciego, controlado con placebo, de diseño factorial $2 \times 2$. Se llevó a cabo en 228 centros de 21 países de distintos continentes con una duración promedio de cinco años. Participaron hombres mayores de 55 años y mujeres mayores de 65 años de diversos grupos étnicos con uno o más factores de riesgo cardiovasculares: perímetro de cintura elevado, bajo nivel de HDL, tabaquismo activo o en los últimos cinco años, disglucemias, historia familiar de enfermedad coronaria prematura y disfunción renal leve. También incluyeron mujeres mayores de 60 años con dos o más de estos factores de riesgo. Los participantes elegidos ingresaron a una fase previa a la aleatorización donde recibieron tratamiento activo con minipolipíldora por cuatro semanas. Luego de esto, 12.705 pacientes que demostraron adherencia y que no tuvieron efectos adversos, fueron aleatorizados a través de una central telefónica gratuita que les asignaba un número perteneciente a cada grupo.

\section{Intervención}

Acorde al diseño $2 \times 2$, quedaron conformados cuatro grupos. Uno recibió rosuvastatina $10 \mathrm{mg} / \mathrm{día}$, otro candesartán $16 \mathrm{mg} /$ día junto con hidroclorotiazida $12,5 \mathrm{mg} / \mathrm{d}$ ía, otro las tres drogas y otro placebo.

\section{Medición de resultados principales}

Se establecieron dos resultados primarios. El primero combinaba muertes de causa cardiovascular, infarto no fatal, ACV no fatal y el segundo incluía los anteriores agregando la revascularización, la falla cardiaca y la resucitación. Los datos fueron analizados por intención de tratar.

\section{Resultados}

Tanto el grupo rosuvastatina como la terapia combinada (candesartán/HCTZ/rosuvastatina) redujeron la incidencia combinada de eventos primarios (ver Tabla 1).

Tabla 1: Resultados primarios del estudio.

\begin{tabular}{|c|c|c|c|}
\hline Desenlaces & $\begin{array}{l}\text { Rosuvastatina } \\
\text { HR (IC 95\%) }\end{array}$ & $\begin{array}{l}\text { Candesartán/HCTZ } \\
\text { HR (IC 95\%)* }\end{array}$ & $\begin{array}{c}\text { Candesartán/HCTZ + } \\
\text { rosuvastatina } \\
\text { HR (IC 95\%) }\end{array}$ \\
\hline $\begin{array}{l}\text { Muerte de causa cardiovascular, infarto no } \\
\text { fatal, ACV no fatal }\end{array}$ & $\begin{array}{c}0,76(0,64 \text { a } 0,91) \\
\text { RRA: } 1,1 \% \text { NNT: } 91\end{array}$ & $0,93(0,79 \text { a } 1,10)^{\star}$ & $\begin{array}{c}\text { 0,71 }(0,56 \text { a } 0,90) \\
\text { RRA: } 1,4 \% \text { NNT: } 72\end{array}$ \\
\hline $\begin{array}{l}\text { Idem anterior agregando: IC, revascularización } \\
\text { y resucitación }\end{array}$ & $\begin{array}{l}0,75(0,64 \text { a } 0,88) \\
\text { RRA: } 1,3 \% \text { NNT: } 77\end{array}$ & $0,95(0,81 \text { a } 1,11)^{\star}$ & $\begin{array}{c}0,72(0,57 \text { a } 0,89) \\
\text { RRA: } 1,6 \% \text { NNT: } 63\end{array}$ \\
\hline
\end{tabular}

HCTZ: hidroclorotiazida. IC: insuficiencia cardíaca. RRA: reducción de riesgo absoluto. NNT: número necesario a tratar. HR: hazard ratio. *No se calcula RRA ni NNT dado que no hubo efecto estadísticamente significativo. ${ }^{*}$ IC 95\%: Intervalo de confianza del 95\%.

Evaluando cada uno de los eventos de manera independiente se evidenció que tanto el infarto no fatal como el ACV no fatal se ven disminuidos con el uso de las estatinas o con la terapia combinada, pero esto no ocurre con la muerte de causa cardiovascular. Si bien el grupo de candesartán/HCTZ no tuvo una incidencia menor de eventos, cuando los resultados fueron analizados en tres subgrupos según los valores de tensión arterial ( $\leq 131,5 \mathrm{mmHg} ; 131,6$ a $143,5 \mathrm{mmHg}$ y $>143,5 \mathrm{mmHg}$ ), se observó en el subgrupo con TAS mayor a $143,5 \mathrm{mmHg}$ una reducción estadísticamente significativa (HR 0,76; IC 95\% 0,60 a 0,96) para los resultados primarios. Los pacientes tratados con estatinas reportaron mayor debilidad muscular, y los tratados con antihipertensivos, mareos, en ambos casos las diferencias de estos efectos adversos con el grupo placebo fue menor al $2 \%$ de forma absoluta.

\section{Conclusión}

La combinación de rosuvastatina 10 mg/día, candesartán (16 mg/día) e HCTZ (12,5 mg/día) [minipolipíldora] se asoció con una reducción significativa de eventos cardiovasculares en personas con riesgo cardiovascular intermedio sin enfermedad cardiovascular establecida.

Fuente de financiamiento: Canadian Institutes of Health Research y AstraZeneca.

\section{Comentario}

El HOPE-3 es un estudio sobre riesgo cardiovascular, que a diferencia de otros estudios sobre el tema, se enfocó en aquellas personas con riesgo cardiovascular intermedio, sin enfermedad cardiovascular establecida (que son el mayor porcentaje de la población con riesgo cardiovascular), incluyó participantes de diferentes etnias y un porcentaje alto de mujeres. Su autor plantea el uso de una minipolipíldora para la prevención primaria de eventos cardiovasculares en pacientes con riesgo cardiovascular intermedio. La minipolipíldora, a diferencia de la polipíldora ${ }^{1,2}$ no contiene aspirina y betabloqueantes. En este trabajo la aspirina no fue incorporada porque demostró tener más efectos adversos que beneficios. Los betabloqueantes se dejaron de lado porque la población que integraba la muestra no necesariamente era hipertensa y de esta manera se habrían verificado mayores efectos adversos como la hipotensión. Este estudio avalaría el uso de estatinas en pacientes de riesgo cardiovascular intermedio. En cuanto al uso de la terapia combinada con antihipertensivos, únicamente aquellos pacientes hipertensos se verían beneficiados. Es importante destacar que siempre debe ser tenido en cuenta el contexto de cada paciente: su riesgo cardiovascular global, otras intervenciones que podrían aplicarse a ese paciente en particular, sus deseos o no de tomar medicación. De esta manera los resultados de este estudio aportan información para la toma de decisiones compartidas con los pacientes.

\section{Conclusiones del comentador}

Si bien el uso de la minipolipíldora (estatinas + antihipertensivo) reduce la incidencia de eventos cardiovasculares, su efecto depende principalmente del efecto de las estatinas. El componente antihipertensivo sería eficaz sólo en hipertensos.

Camila Volij [ Servicio de Medicina Familiar y Comunitaria del Hospital Italiano de Buenos Aires camila.volij@ hospitalitaliano.org.ar ]

Volij C. HOPE-3: ¿Minipolipildora para la prevención primaria de eventos cardiovasculares en personas de riesgo intermedio? Evid Act Pract Ambul 2016;19(4):114.Comentado de: Yusuf S. Cholesterol Lowering in Intermediate-Risk Persons without Cardiovascular Disease. N Engl J Med 2016; 374:2021-2031. PMID: 27040132.

\section{Referencias}

1. Yusuf S y col. Effects of a polypill (polycap) on risk factors in middle-aged individuals without cardiovascular disease (TIPS): a phase Il, double-blind, randomised trial. Lancet 2009 Apr 18; $373,1341-1351$. 2. NJ, Law MR. A strategy to reduce cardiovascular disease by more than $80 \%$. BMJ 2003;326:1419. 